\title{
Features of Structure of Electrodeposited Metals Resulting from Exposure to External Force Parallel, Normal or Inclined to the Crystallization Front
}

\author{
Oleg B. Girin \\ Department of the Materials Science, Ukrainian State University of Chemical Technology, Dnipropetrovsk, Ukraine
}

Email address:

girin@ua.fm

To cite this article:

Oleg B. Girin. Features of Structure of Electrodeposited Metals Resulting from Exposure to External Force Parallel, Normal or Inclined to the Crystallization Front. Advances in Materials. Special Issue: Advances in Electrodeposited Materials: Phase Formation, Structure and Properties. Vol. 4, No. 3-1, 2015, pp. 1-14. doi: 10.11648/j.am.s.2015040301.11

\begin{abstract}
To prove whether phase formation in an electrochemically deposited metal indeed passes a liquid state stage, experiments involving application of an external force during electrodeposition were carried out and structural features of deposit were subsequently determined. Following experimental facts in support of the phenomenon in point were observed: (1) occurrence of anisotropic pattern of electrodeposits of metals being electrodeposited, smoothing out of deposits surface morphology and reduction in their surface roughness arising from exposure to a minor external force parallel to the crystallization front; (2) refinement of intracrystalline structure and of surface morphology of metals being electrodeposited under exposure to a minor external force normal to the crystallization front; (3) dislocation density rise in metals being electrodeposited under exposure of a minor external force normal to the crystallization front; (4) surface layers plastic deformation in metals being electrodeposited by solid particles travelling due to action of minor external force inclined to the crystallization front; and (5) occurrence of indents from solid particles representing the particles' shape and morphology at sites of the particles' separation from deposit surface in metals being electrodeposited under exposure to minor external force inclined to the crystallization front.
\end{abstract}

Keywords: Structure, Surface Morphology, Dislocation Density, Electrodeposited Metal, Liquid State, External Force

\section{Introduction}

According to current views, phase formation in a metal being electrodeposited occurs by "incorporation" or "embedding" of ions from the water solution or of atoms (adatoms or adions) evolved at deposit surface into the deposit crystal lattice. The existing concepts only differ on the issue of ion discharge location on the surface of growing deposit. One approach sees ion discharge take place predominantly at the "growth site" directly, i. e. at the point where the ion is incorporated into the deposit lattice [1-4]. The other concept views a predominant ion discharge as occurring at random sites of the cathode (deposit) surface; the atom is then allegedly delivered by surface diffusion to the "growth site" [5-8].

Although put forward half a century ago, neither of the two concepts has been satisfactorily supported experimentally. Indeed, no answers have been given to the questions of how a metal ion located in an amorphous liquid phase of one substance, namely in the water solution, is directly incorporated into a solid crystalline phase of a different substance, i. e. metal being electrodeposited, and what phase transitions and structural changes in the deposit accompany this process.

Moreover, it is still unclear in what aggregate state the metal being electrodeposited is while its atoms diffuse long distances to "growth sites" at ambient temperature or what values of the activation energy and diffusivity are characteristic of such low temperature diffusion. In spite of both concepts being hypothetical, they found entry into recent monographs on phase formation in metals during electrodeposition as if they were undisputable scientific facts [9-12].

Importantly, a multitude of scientific findings have been accumulated by now, like the formation of intermediate modifications in polymorphous metals being electrodeposited, the formation of intermediate phases in metal alloys being electrodeposited, and occurrence of abnormally high vacancy 
concentrations in electrodeposited metals, which are difficult to explain satisfactory using current views on phase formation of metals being electrodeposited.

Besides, the current views on phase formation and structure development in metals during electrodeposition are contradicted by experimental evidence - density rise in metals electrodeposited under exposure to a minor external force normal to the crystallization front, wavelike flow in surface layers of metals being electrodeposited under exposure to a minor external force parallel to the crystallization front, or eutectic formation during simultaneous electrodeposition of two metals, to name just a few.

Therefore, existing views on phase formation in metals during electrodeposition need to be elaborated and further developed based on an alternative platform, involving advancing and realization of new ideas and approaches.

A previously unknown phenomenon of phase formation via a liquid state stage in metals being electrodeposited was recently established by synthesis of experimental and theoretical findings [13-26]. Essentially, this phenomenon is to be understood as follows: during metal electrodeposition onto a solid cathode in an aqueous environment, a deeply undercooled metal liquid evolves in the form of multiple liquid atomic clusters avalanching at various sites near the cathode or the growing deposit and solidifies ultra rapidly at the deposition temperature to appear in a crystalline, amorphous or quasi-crystalline phase [13-16].

This phenomenon is due to an extremely rapid, burst-like liberation of metal resulting from a chain reaction of electrochemical evolution of atoms and transition of the atomic clusters from a liquid to a more stable, solid state [15].

Following experimental facts prove the existence of the phenomenon of phase formation via a liquid state stage in metals being electrodeposited:

- (1) occurrence of a diffraction pattern typical of an amorphous state during electrochemical deposition of metal, the pattern then changing to one typical of a crystalline state without supplying heat [13-15];

- (2) density rise, accompanied by declined porosity together with decreased X-ray diffraction intensity, in metals being electrodeposited under exposure to a minor external force normal to the crystallization front $[17,18]$;

- (3) wavelike flow in surface layers of metal being electrodeposited under exposure to a minor external force parallel to the crystallization front, involving wave bending around mechanical obstacles and wave-crest foaming [18, 19];

- (4) deposits shape change in metals being electrodeposited under exposure to a minor external force parallel to the crystallization front, like thickening, alteration of form or extension in force direction [18, 19];

- (5) preferential development of metal deposits beyond cathode edge in the direction of the minor external force parallel to the crystallization front during electrodeposition [19];

- (6) texture development suppression even to the point of complete disordering of grain lattice preferred orientations during electrodeposition under exposure to a minor external force opposite to texture axis, as opposed to texture development facilitation by exposure to a similar force acting along the texture axis [20];

- (7) formation of amorphous structure typical of "frozen" liquid metal due to electrodeposition under conditions preventing crystallization [21];

- (8) occurrence of defect-rich crystal structure in metals being electrodeposited, similar to the one of metals quenched from liquid state [21, 22];

- (9) formation of intermediate modifications, typical of quenched polymorphous metals from liquid state, in polymorphous metals being electrodeposited [22, 23];

- (10) formation of intermetallics in electrodeposited binary alloys of metals differing in valence [24];

- (11) formation of carbides or hydrides in electrodeposited transition metals alloyed with carbon or hydrogen respectively [24];

\section{4];}

(12) eutectic formation in binary system electrodeposition

- (13) interdiffusion of atoms of cathode metal and metal to be electrodeposited at room temperature [25];

- (14) formation of chemical compounds at interface between solid metal cathode and metal being electrodeposited $[23,25]$.

The existence of the phenomenon of phase formation via a liquid state stage in metals being electrodeposited is supported by the following experimental findings:

- (1) developing of structural state in metals being electrodeposited, having all features typical of metals solidified from a deeply undercooled metallic liquid [21, 23, 25];

- (2) occurrence of porous structure in metals being electrodeposited which exhibits all features characteristic of that in metals upon solidification from liquid state in a hydrogen-saturated environment [26];

- (3) crystallization of polymorphous metal being electrodeposited in a form of intermediate modification identical to the intermediate modification in this metal upon its solidification from liquid state in a hydrogen-saturated environment [26];

- (4) increasing porosity in metal with increased dissolution of hydrogen into it during electrodeposition [26].

The phenomenon in point is manifested in consistent changes of substructure [16], surface morphology [16, 21], structural state $[16,22]$, crystallographic texture $[16,23]$ and structural nonhomogeneity $[23,25]$ in metals with deeper undercooling during electrodeposition.

The aim of this work was the further experimental verification of the existence of the discovered phenomenon based on analysis of structural features in metals electrodeposited under exposure to an external force parallel, normal or inclined to the crystallization front.

The general idea of this study was that the fundamental distinction between a metal's solid and liquid state consists in the difference in the metal's respective capacity to change its shape and structure when exposed to a relatively small external force. With a solid metal, forming requires action of 
heavy forces (as in forging, press forming or rolling) and exposure to a minor external force will be to no effect; contrastingly, changing the form of the same metal in a liquid state will be possible with a relatively small load. Furthermore, application of a minor force to a metal in a solid state will not bring about changes in its structure on such a dramatic scale as in this metal subjected to a similar force while solidifying from a liquid state.

If phase formation in a metal being electrodeposited indeed passes a liquid state stage, then exposure of the emerging electrodeposit to a minor external force, like centrifugal one, may be expected to give rise to structural features typical of liquid metal subjected to some force while solidifying. In this context, structural features of electrodeposited metals typical of liquid metal solidified in the presence of external force will serve as a proof of the existence of the phenomenon in point.

\section{Experimental Proof of the Phenomenon's Existence}

\subsection{Idea One and Its Realization}

It is well-known that a major distinction between a solid and a liquid state of a metal lies in their flow ability [27]. The flow ability of a body may be defined as its deformation rate if subjected to a static shear load. For viscosity as flow characteristic of a metal, the difference between the solid and the liquid state is of the order of $10^{20}$ [27].

If a liquid state stage is passed in phase formation of a metal during electrodeposition, one can expect changes in the pattern, surface morphology and roughness of the deposit when a minor external force is applied parallel to the crystallization front. Consequently, the high flow ability of metallic liquid that permanently renews in microareas near the metal deposit surface while being exposed to an external force should result in some special features of the deposit. First, the final deposit pattern should be anisotropic and elongated in the direction of the force. Second, the deposit surface morphology is expected to be more smooth while the surface roughness should be lower compared to that of a deposit evolved in the absence of such a force.

The occurrence of deposit with an anisotropic pattern, a smoothed-out surface morphology and a lower surface roughness due to a minor external force acting parallel to the crystallization front will be a proof of the existence of the phenomenon in point.

\subsubsection{Materials and Methods}

A centrifugal setup described earlier [28] was used to exert a minor external force parallel to the crystallization front on the metal being electrodeposited. In this setup, direct current was applied to rotating electrochemical cells in the centrifuge; the setup control unit was provided with a rotational frequency sensor. The setup provided action of the centrifugal force proportional to the normal acceleration (or overload) $\mathrm{kg}, \mathrm{k}$ being the overload coefficient, between $1 \mathrm{~g}$ and $1256 \mathrm{~g}$ by changing the rotational frequency of the rotor from 0 to 3000 rpm [28].

To verify the above idea, special electrochemical cells were made that had a partition placed between the cathode and the anode and having a horizontal slot in its upper portion (Fig. 1). This slotted partition between the anode and the cathode, together with an electrolyte of low dispersive capacity, provided for formation of a small sized cathode deposit in the tests.
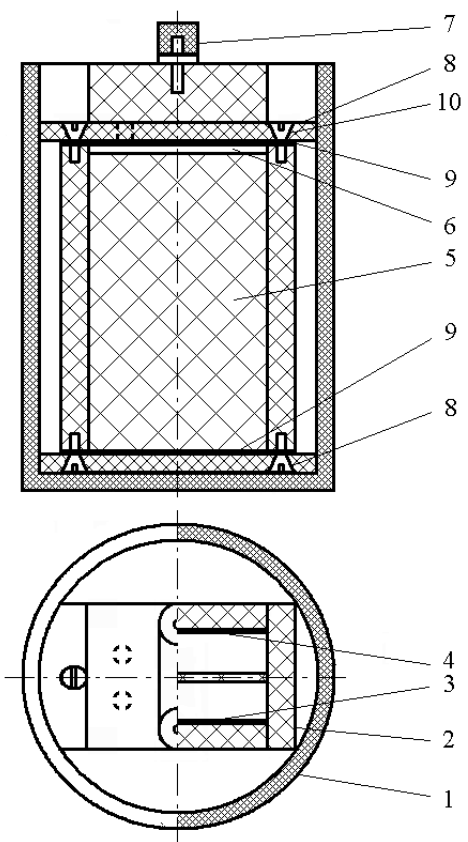

Figure 1. Schematic of electrochemical cell with a slotted partition between the cathode and the anode: 1 -centrifuge holder; 2 - cell; 3 -cathode; 4 anode; 5 -partition; 6 -slot; 7 -current lead clamps; 8 -cell lid; 9 -rubber sealings; 10 - fixing screws.

The cell design eliminated any possibility of electrolyte flow relative to the cathode during electrodeposition. In centrifuge operation, the cell was rotated together with the centrifuge rotor and preserved horizontal position. The cathode was attached to the cell wall in parallel relation to the cell axis, so it remained parallel to the rotor radius during cell rotation. Since the centrifugal force is always directed along the rotor radius from the centre of rotation, such arrangement of the cathode ensured application of an external force parallel to the crystallization front to the metal being electrodeposited.

Zinc was selected as the model metal to be electrochemically deposited from a solution containing 450 $\mathrm{g} / 1 \mathrm{ZnSO}{ }_{4} \cdot 7 \mathrm{H}_{2} \mathrm{O}$ and $30 \mathrm{~g} / 1 \mathrm{Al}_{2}\left(\mathrm{SO}_{4}\right)_{3} \cdot 18 \mathrm{H}_{2} \mathrm{O}$. The electrolyte choice was dictated by its low dissipative capacity of only $24 \%$ [29]. This cited value of $24 \%$ was confirmed in the electrolyte tests using a Haring-Blum cell at a current density of 8.0 $\mathrm{A} / \mathrm{dm}^{2}$, electrolyte acidity of $4.0 \mathrm{pH}$ and a temperature of $20{ }^{\circ} \mathrm{C}$. Accordingly, this mode of electrodeposition was employed for the tests on zinc as being in full agreement with the conditions for the realization of the above idea. The electrode dimensions and the cell volume were such that the volume current density did not exceed $1 \mathrm{~A} / 1$ to avoid 
electrolyte heating during electrodeposition.

The deposit pattern and its surface morphology were investigated using secondary electron images from a REhM-106I scanning electron microscope. The deposit surface roughness was measured with a Type 252 recording profilometer to yield $R_{a}$ values and surface profilograms at magnifications of $\times 50$ (horizontal) and $\times 200$ (vertical).

\subsubsection{Results and Discussion}

Tests revealed that zinc deposits obtained in the low dissipative electrolyte under ordinary conditions, i. e. at $\mathrm{k}=1$, displayed an ellipsoidal pattern. Fig. 2a shows an ellipsoidal deposit slightly extended horizontally in the direction of the horizontal slot in the cathode-anode partition. This was a predictable feature determined by the experimental conditions.

A characteristic feature displayed by zinc electrodeposited in the absence of an external force was an inhomogeneous surface morphology. The deposit central area thus consisted of globular formations having a branched petal-like dendritic morphology (Fig. 2b). As distinct from the central area, the peripheral portions had a less coarse morphology. It should be noted that the central and peripheral areas were similar in their morphology.

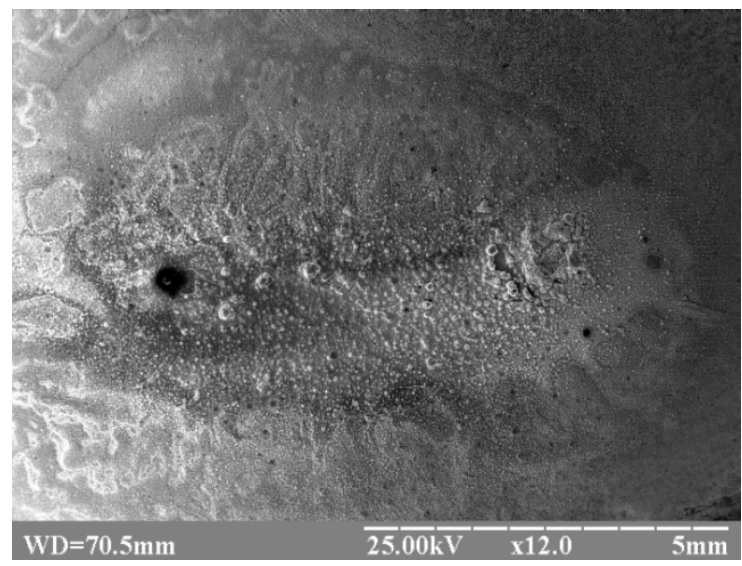

(a)

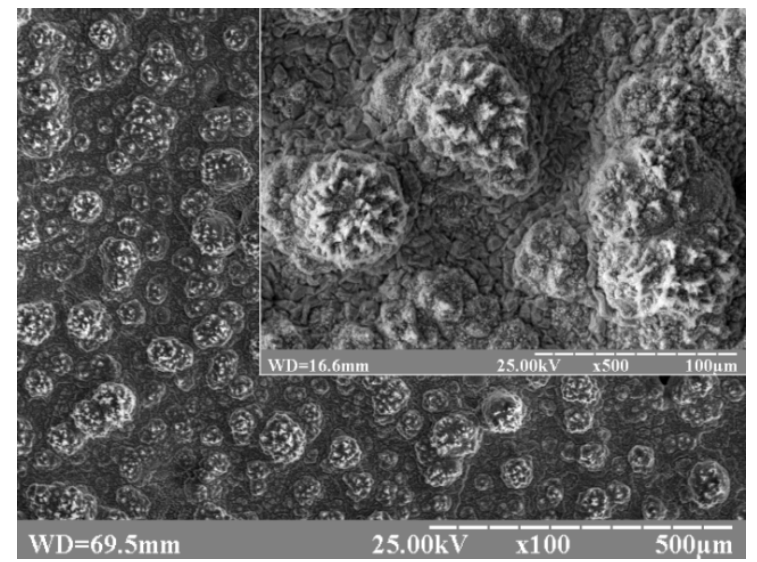

(b)

Figure 2. SEM images of the deposit of electrodeposited zinc obtained at ordinary conditions $(k=1)$ : (a) general appearance; (b) surface morphology in deposit central area.
Surface roughness measurements on various deposit areas revealed that the central area was less smooth than the periphery. Thus an average $R_{a}$ value in the central area was $3.75 \mu \mathrm{m}$, i. e. about two times greater than in the periphery $(2.01 \mu \mathrm{m})$. Similarly, profilograms of the respective deposit portions showed that the roughness was greater in the deposit centre (Fig. 3a) than in its periphery, Fig. 3b.

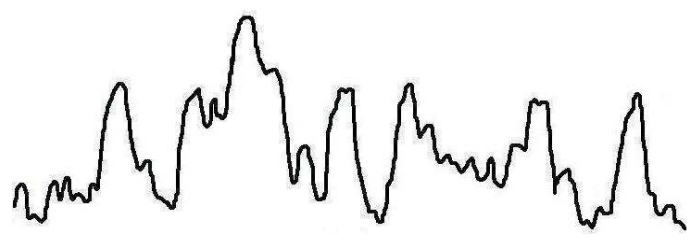

(a)

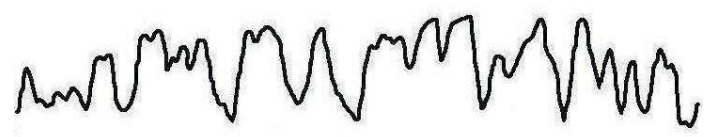

(b)

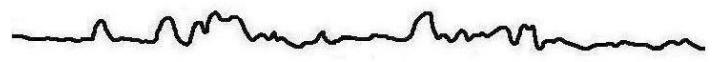

(c)

Figure 3. Profilograms of zinc deposits: (a) central area of deposit obtained under ordinary conditions; (b) peripheral area of deposit obtained under ordinary conditions; (c) deposit obtained under external force action during electrodeposition.

An action of a minor external force parallel to the crystallization front during electrodeposition of zinc resulted in a considerable reduction in the deposit surface roughness. The $R_{a}$ was at just $0.58 \mu \mathrm{m}$ on the average and, notably, the surface roughness was practically uniform over the entire area while the profilograms were smoothed out too, see Fig. 3c.

As can be seen in Fig. 4a, zinc electrodeposition under exposure to an external force parallel to the crystallization front resulted in an anisotropic pattern of the deposit extended in the force direction. The surface morphology in such cases featured smoothed-out shapes with relatively gently inclined edges, Fig. 4b. A distinctive feature of the deposits precipitated under exposure to an external force was uniform surface morphology over the entire area.

The effects of external force action displayed as the changes in deposit pattern, morphology and roughness were confirmed in tests on electrodeposition of cadmium from a sulphuric electrolyte with a low dissipative capacity of $28 \%$.

The occurrence of an anisotropic deposit pattern aligned with the external force, the smoothing-out of the deposit surface morphology and the reduction in the surface roughness due to electrodeposition under exposure to an external force can be explained using the proposed concept of passing a liquid state stage by the metal being electrodeposited [13-16]. Indeed, due to an extremely rapid nature of a burst-like growth event (about $10^{-7} \mathrm{~s}$ ) generating an average of 40 to 60 atoms [15], these have no time to build a long range ordered structure. The multitude of liquid atomic clusters 
avalanching at various sites near the cathode or growing deposit surface constitute a highly undercooled liquid phase of the metal being electrodeposited [15].

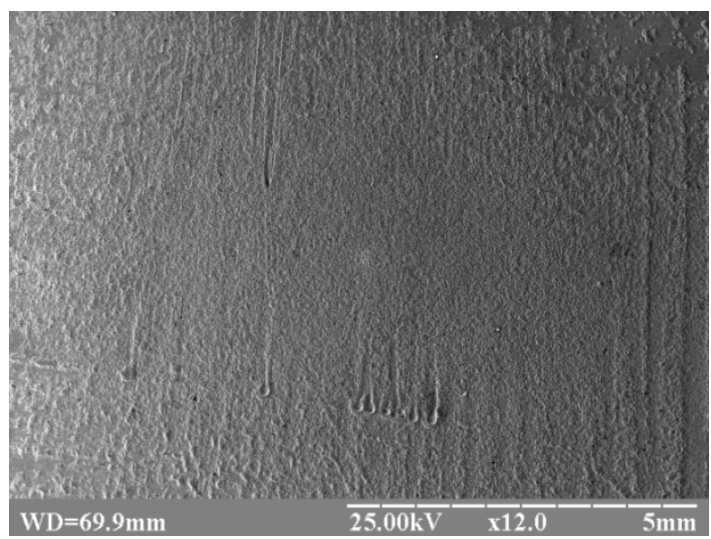

(a)

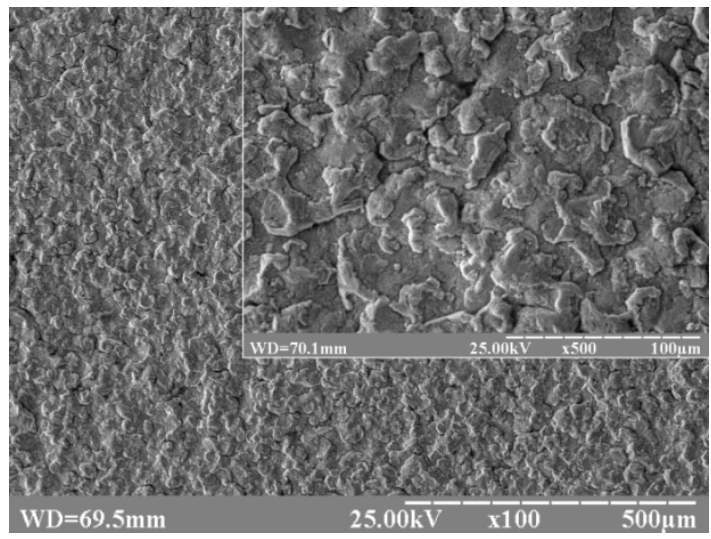

(b)

Figure 4. SEM images of the deposit of electrodeposited zinc obtained under exposure to an external force parallel to the crystallization front $(k=1256)$ : (a) general appearance; (b) surface morphology; force direction top to bottom.

Since such atomic clusters evolve continually during metal electrodeposition, they coalesce to make larger bodies that are deformed and move in the force direction. Upon their extremely rapid solidification, these bodies of atoms build up an anisotropic pattern of the electrodeposit having a smoothed-out morphology and a reduced surface roughness.

However, under assumption that phase formation in a metal being electrodeposited does occur by its lattice "embedding" ions from the aqueous solution or atoms evolved at its surface [1-8], none of the effects observed could have taken place. The reason is that the external force action represented here by the centrifugal force is clearly insufficient to produce plastic strain in solid surface layers of the electrodeposited metal.

Therefore, the occurrence of an anisotropic pattern of electrodeposited metal, the smoothing-out of deposit surface morphology and the reduction in its surface roughness due to a minor external force parallel to the crystallization front all prove the phenomenon's existence, namely phase formation via a liquid state stage in metals being electrodeposited.

\subsection{Idea Two and Its Realization}

It is well-known that action of an external force normal to the solidification front of a metallic liquid, which is typical of what is called die-casting, leads to a drastic refinement of the resulting solid metal structure [30-32]. One may therefore expect that exposure of the metal being electrodeposited to a minor external force normal to the crystallization front will give rise to some refinement of intracrystalline structure and of grain aggregates that define surface morphology of the deposit.

In this context, refinement of intracrystalline structure in metal being electrodeposited under action of a minor external force normal to the crystallization front, together with a finer deposit surface morphology, will demonstrate the existence of the phenomenon in point.

\subsubsection{Materials and Methods}

Based on consideration that the inferred effects should be manifested even at minor values of the external action, centrifugal force was employed same as in the above experiments. The overload was varied stepwise between $1 \mathrm{~g}$ and $1256 \mathrm{~g}$ by changing the rotor rotational speed from 0 to $3000 \mathrm{rpm}$. In order to realize idea two, electrochemical cells were provided that excluded any possibility of the electrolyte movement with respect to the cathode during metal electrodeposition (Fig. 5).

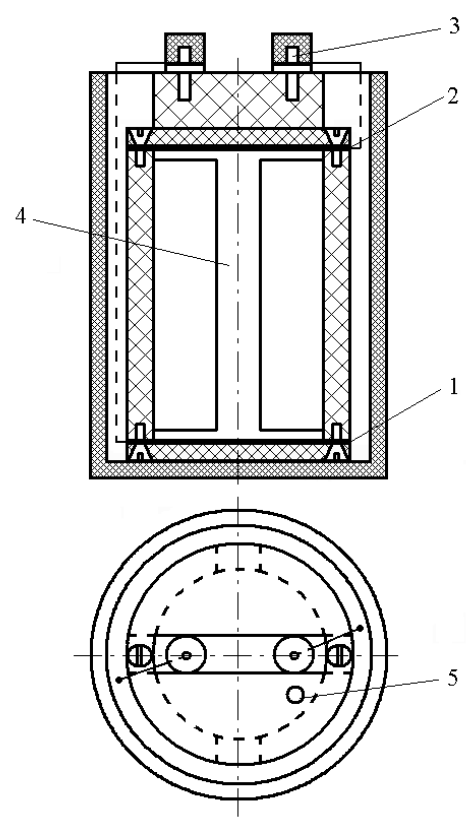

Figure 5. Schematic of electrochemical cell: 1 - cathode; 2 - anode; 3 current lead; 4 - reinforcement rib; 5 -opening for feeding electrolyte.

In operation of the centrifuge, the cells remained in a horizontal position and rotated jointly with the rotor. The cathode was attached to the cell bottom perpendicularly to the cell axis, see Fig. 5; accordingly, the cathode was perpendicular to rotor radius during rotation of the cell. Since the centrifugal force developing in rotation is always directed radially outwardly from the rotor centre, this position of the 
cathode ensured that a force normal to the crystallization front acted on the metal being deposited. The distance between the centrifuge rotation axis and the cathode surface in the cell, i. e. radius of gyration, was equal to $12.5 \cdot 10^{-2} \mathrm{~m}$ while the inter-electrode distance was at $5 \cdot 10^{-2} \mathrm{~m}$.
To avoid hydrogen effects on structure of deposits precipitating in the field of centrifugal force, copper and nickel were electrodeposited at yields of metal ranging from 99.5 to $100 \%$ (Table 1 ).

Table 1. Electrolytes composition and parameters of electrodeposition of metals.

\begin{tabular}{|c|c|c|c|c|}
\hline Metal & Electrolytes Composition, $\mathrm{g} \mathrm{L}^{-1}$ & Electrolyte Temperature, ${ }^{\circ} \mathrm{C}$ & Current Density, $\mathrm{A} \mathrm{dm}^{-2}$ & Current Yield, \% \\
\hline $\mathrm{Cu}$ & $\begin{array}{l}\mathrm{CuSO}_{4} \cdot 5 \mathrm{H}_{2} \mathrm{O}-250 \\
\mathrm{H}_{2} \mathrm{SO}_{4}-50\end{array}$ & 25 & 1 & 100 \\
\hline $\mathrm{Ni}$ & $\begin{array}{l}\mathrm{NiSO}_{4} \cdot 7 \mathrm{H}_{2} \mathrm{O}-330 \\
\mathrm{NiCl}_{2} \cdot 6 \mathrm{H}_{2} \mathrm{O}-50 \\
\mathrm{H}_{3} \mathrm{BO}_{3}-35 \\
\mathrm{C}_{11} \mathrm{H}_{23} \mathrm{COONa}-0.1\end{array}$ & 25 & 1 & 99.5 \\
\hline
\end{tabular}

Specimen deposition time was kept at $60 \mathrm{~min}$. To prevent metal ion depletion in electrolyte during tests, soluble anodes were used. Pitting arising from prolonged electrodeposition of nickel was avoided by $0.1 \mathrm{~g} / 1$ sodium lauryl sulphate addition to the electrolyte. Cathodes were in the form of thin plates of nickel (for copper deposition) or copper (for nickel).

The intracrystalline structure in the electrodeposits was characterized by size of subgrains (or mosaic blocks). This structural characteristic was selected because metal subgrains are those grain areas that feature an ideal crystal lattice and are thus defined as regions with parallel lattice planes. Therefore, even a minimal structural refinement in a metal will be manifested first of all in a reduced subgrain size.

It was found in preliminary experiments that crystallographic texture in copper and nickel electrodeposited under conditions involved is of axial type [110] and $[210]+[221]$ respectively [20]. With due consideration of structural inhomogeneity effects in oriented electrodeposited metals [33-35], the size measurements were performed on subgrains of the orientationally disordered component of texture.

Diffraction experiments were carried out using an upgraded DRON-3 X-ray diffractometer $\left(\mathrm{Cu}-\mathrm{K}_{\alpha}, \mathrm{U}=30 \mathrm{kV}, \mathrm{I}=20 \mathrm{~mA}\right)$ and a technique described earlier [36]. Subgrain sizes in orientationally disordered grains were obtained from diffraction patterns of specimens placed in the holder with the texture axis perpendicular to the goniometer axis [36]. In doing so, the method of diffraction peak shape approximation for two orders of reflection from plane $\{111\}$ was used. Reference specimens displaying no effects of texture or extinction were prepared from copper or nickel powder compacted and annealed to a monolithic condition.

Surface morphology of electrodeposits was characterized using a scanning electron microscope REhM-106I and secondary electron imaging. The ultimate pressure in the SEM column in the vicinity of the electron gun did not exceed $6.7 \cdot 10^{-4} \mathrm{~Pa}$, the gun current was $98 \mathrm{~mA}$.

\subsubsection{Results and Discussion}

The experiments revealed that metals electrodeposited under exposure to a minor external force normal to the crystallization front displayed a refined structure. Thus Fig. 6 shows that increasing overload from $1 \mathrm{~g}$ to $1256 \mathrm{~g}$ in copper being electrodeposited was accompanied by a considerable drop in intensity of X-ray diffraction peaks (111) and (222) while peaks broadening showed an increase.

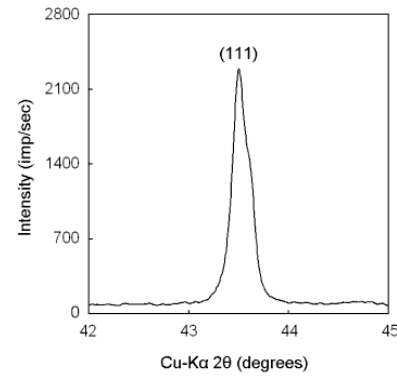

(a)

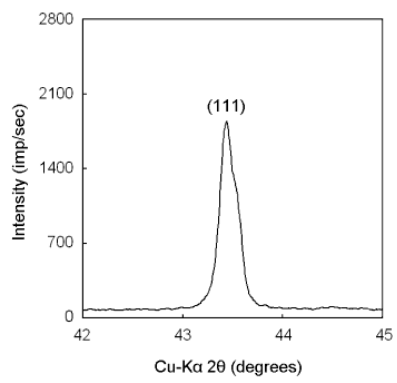

(c)

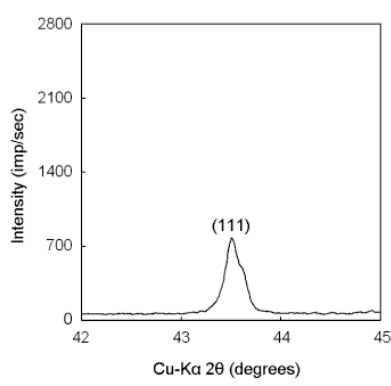

(e)

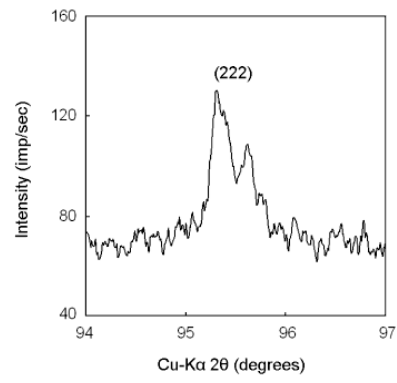

(b)

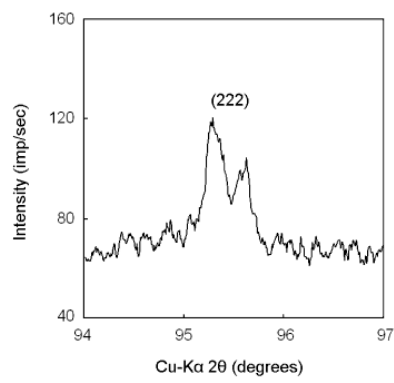

(d)

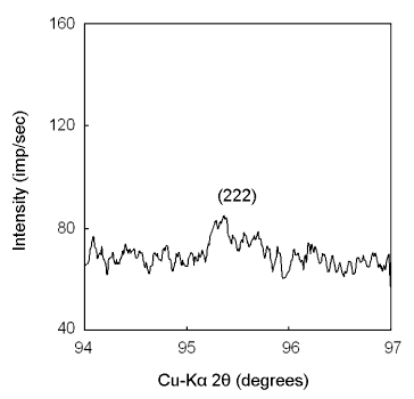

(f)
Figure 6. Intensity curves for X-ray diffraction peaks $\{111\}(a, c, e)$ and $\{222\}$ $(b, d, f)$ in copper electrodeposited under exposure to external force normal to the crystallization front by overload of $1 g(a, b), 35 g(c, d)$ and $1256 g(e, f)$.

The intensity drop and the broadening increase alike were observed on an appreciable scale even at the minimum 
overload step of $35 \mathrm{~g}$ corresponding to a rotational speed of only $500 \mathrm{rpm}$ (Fig. 6c and 6d). Further increasing overload to $1256 \mathrm{~g}$ (3000 rpm) was accompanied by additional peak intensity weakening and peak broadening (Fig. 6e and 6f).

The measurements on copper deposits revealed an appreciable reduction of subgrain size from 388 to $246 \mathrm{~nm}$ due to increasing overload from $1 \mathrm{~g}$ to $35 \mathrm{~g}$. An additional overload rise to $1256 \mathrm{~g}$ was accompanied by further subgrain refining down to $93 \mathrm{~nm}$.

Parallel to intracrystalline structure refining in copper electrodeposited under external force action, deposit surface morphology change was observed (Fig. 7).

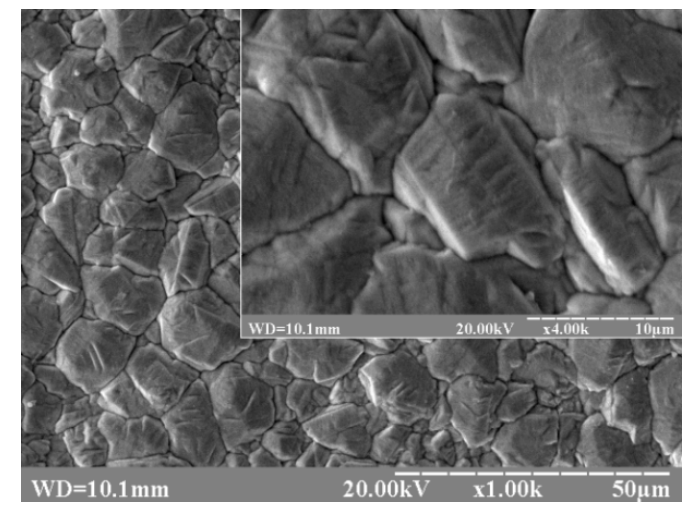

(a)

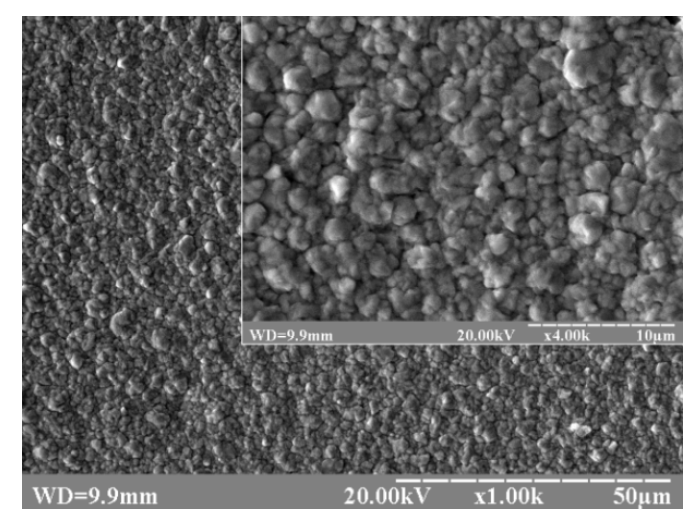

(b)

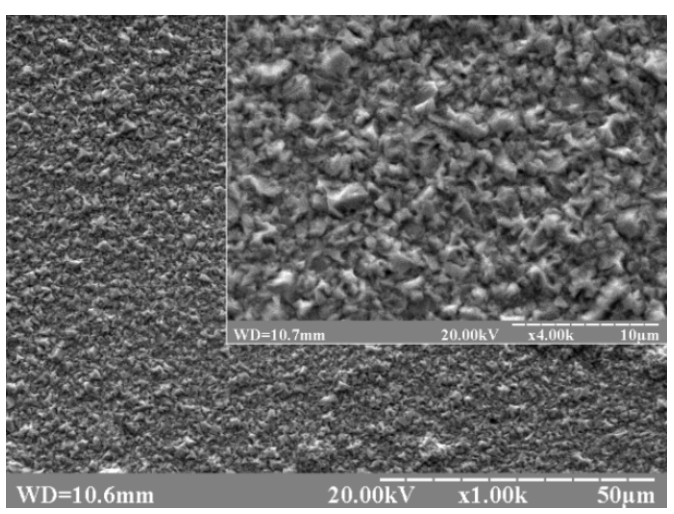

(c)

Figure 7. SEM images of copper electrodeposits precipitated under exposure to external force varied in proportion to overload changed from $1 \mathrm{~g}$ ( $a$, initial condition) to $35 \mathrm{~g}$ (b) and to $1256 \mathrm{~g}(\mathrm{c})$. The force was applied normal to the crystallization front.
Compared to $1 \mathrm{~g}$ when the copper surface displayed faceted shapes and grain aggregates size averaged at about $10 \mu \mathrm{m}$ (Fig. 7a) application of a minor additional force corresponding to $35 \mathrm{~g}$ during electrodeposition led to refinement of surface formations by approximately an order of magnitude accompanied by manifestations of spherical shape (Fig. 7b). Subsequent increasing of the overload to a maximum value of $1256 \mathrm{~g}$ resulted in additional refinement of the structure and occurrence of heavily deformed shapeless formations on the surface (Fig. 7c).

With nickel electrodeposits, similar structural change was observed as overload and the resulting external force were increased (Fig. 8).

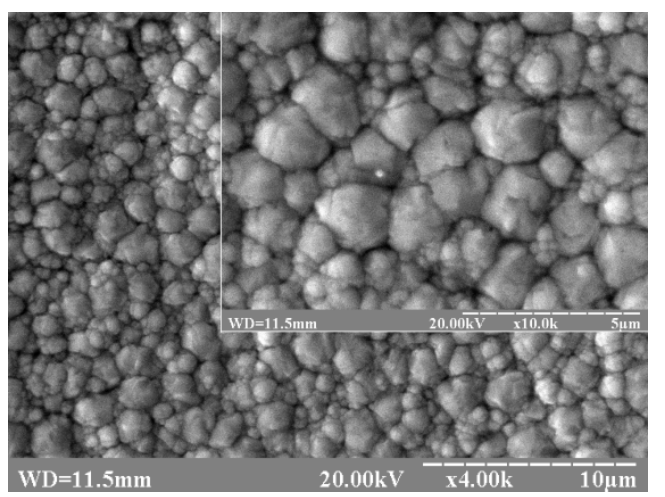

(a)

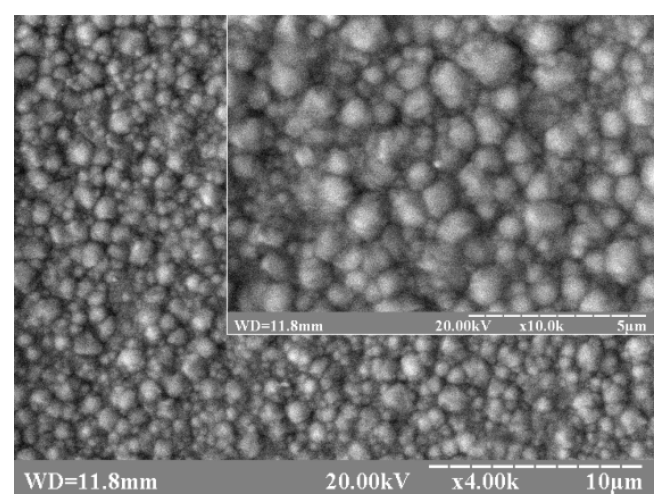

(b)

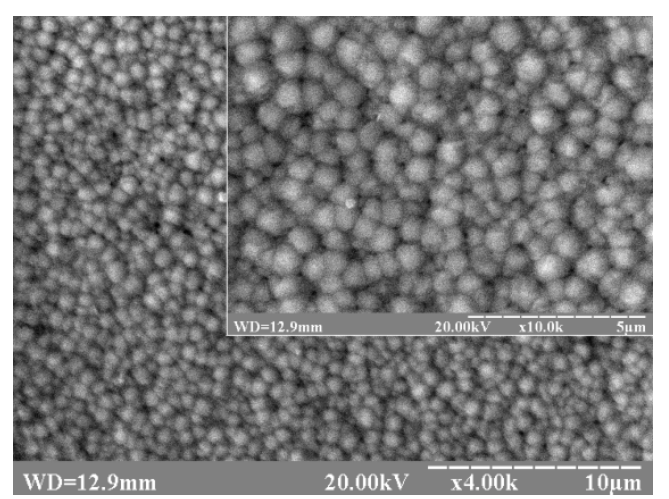

(c)

Figure 8. SEM images of nickel electrodeposits precipitated under exposure to external force varied in proportion to overload changed from $1 \mathrm{~g}$ (a, initial condition) to $35 \mathrm{~g}(\mathrm{~b})$ and to $1256 \mathrm{~g}$ (c). The force was applied normal to the crystallization front. 
As can be seen from Fig. 8, raising external force normal to crystallization front indeed led to a finer surface morphology of nickel deposits. Notably, the most dramatic reduction in surface formation size was measured at a minimum additional overload of only $35 \mathrm{~g}$ (Fig. 8b). Further increasing of external force by an overload of up to $1256 \mathrm{~g}$ was accompanied by just a negligible refinement of grain aggregates (Fig. 8c).

The findings on surface morphology features of nickel electrodeposits obtained during application of external force normal to crystallization front are confirmed by experimental data on their intracrystalline structure. As seen from Fig. 9, raising the external force applied in electrodeposition of nickel led to (111) и (222) X-ray diffraction peaks broadening and to their intensity weakening. Notably, the broadening and weakening effects were distinct even at a minimal additional overload of $35 \mathrm{~g}$.

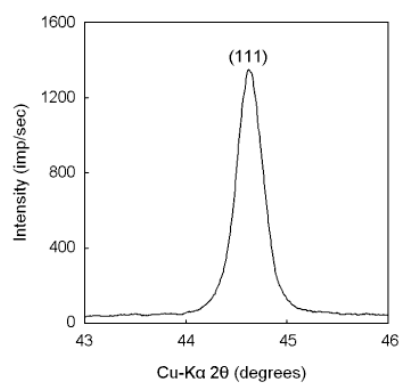

(a)

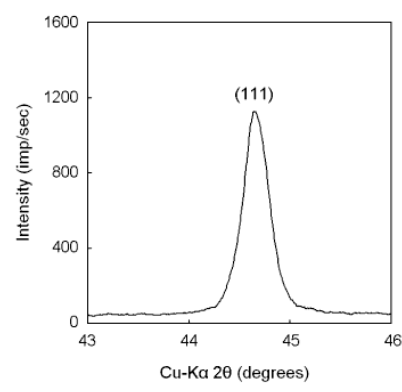

(c)

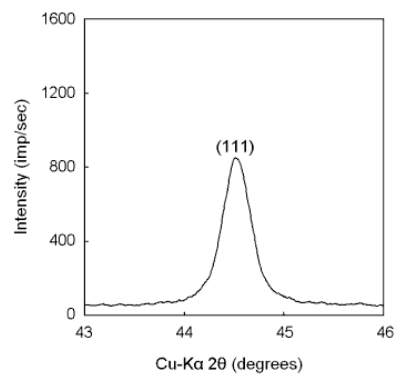

(e)

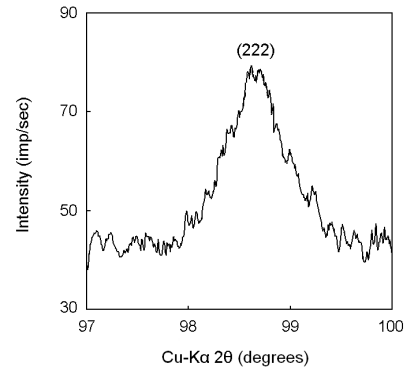

(b)

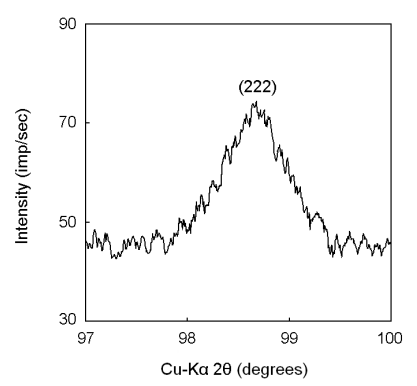

(d)

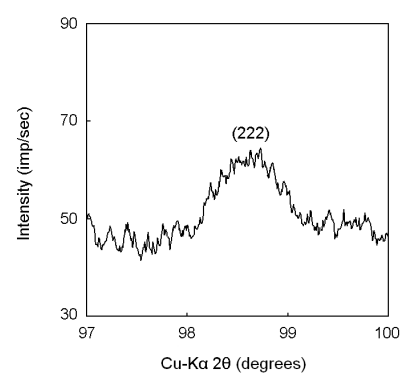

(f)
Figure 9. Intensity curves for X-ray diffraction peaks $\{111\}(a, c, e)$ and $\{222\}$ $(b, d, f)$ in nickel electrodeposited under exposure to external force normal to the crystallization front by overload of $1 \mathrm{~g}(a, b), 35 \mathrm{~g}(c, d)$ and $1256 \mathrm{~g}(e, f)$.

The experiments showed that the refinement of intracrystalline structure in nickel deposits was most pronounced (subgrain size drop from 96 to $67 \mathrm{~nm}$ ) after raising overload from $1 \mathrm{~g}$ to $35 \mathrm{~g}$. Further increasing the overload even to $1256 \mathrm{~g}$, although accompanied by structure refinement too, resulted in subgrain size reduction by $20 \%$ at most.

It should be noted that the above results are in good agreement with data $[37,38]$ which likewise revealed refinement of intracrystalline structure [37] and surface morphology [38] in electrodeposited nickel due to increased overload and, consequently, greater centrifugal force normal to crystallization front. The fact that nickel electrodeposited under centrifugal force action developed a fine grain structure was then explained by an enhanced ion transport to the cathode surface presumably leading to accelerated nucleation [38]. This structure refining mechanism, however, seems unlikely for metals being electrodeposited, because effects of centrifugal force on a metal ion in electrolyte are negligible due to the ion's minute mass.

Let us assume that no intermediate liquid phase of the metal contributes to its structural refinement during electrodeposition involving an external force. Starting from this assumption, let us estimate how high an external force must be to refine structure in a metal being electrodeposited when it is in a solid state. It is well-known that in order to refine structure of a solid metallic material, the load applied must be high enough to arouse compressive stress sufficient to give rise to a plastic deformation in it. The stress needed for permanent deformation to occur is known as yield strength [39]. Its value is $62 \mathrm{MPa}$ for copper and $83 \mathrm{MPa}$ for nickel [40].

Let us find compressive stress $\mathrm{S}$ giving rise to structure refinement in a metal being electrodeposited under application of an external (here, centrifugal) force. A simple manipulation leads to

$$
\mathrm{S}=\mathrm{d} \cdot \mathrm{h} \cdot \mathrm{k} \cdot \mathrm{g}
$$

$\mathrm{d}$ being electrolyte density, $\mathrm{kg} / \mathrm{m}^{3}$; h electrolyte head above the deposit, $\mathrm{m}$; $\mathrm{k}$ overload coefficient; $\mathrm{g}$ acceleration of gravity equal to $9.81 \mathrm{~m} / \mathrm{s}^{2}$.

The values of $\mathrm{d}$ for the electrolytes used here for copper and nickel deposition are $1.165 \cdot 10^{3}$ and $1.215 \cdot 10^{3} \mathrm{~kg} / \mathrm{m}^{3}$ respectively. The respective values of $\mathrm{h}$ and $\mathrm{k}$ are $5 \cdot 10^{-2} \mathrm{~m}$ and 35. Substituting these values in (1) gives $\mathrm{S}=0.020 \mathrm{MPa}$ for copper being electrodeposited and $\mathrm{S}=0.021 \mathrm{MPa}$ for nickel being electrodeposited. These results are incommensurable with the yield strength values for copper and nickel.

Even for the maximum experimental overload coefficient $\mathrm{k}$ $=1256$, respective stress developed by centrifugal force in copper being electrodeposited or nickel will be 0.717 and $0.748 \mathrm{MPa}$. Both make just one per cent of the yield strength values cited and are clearly not sufficient to give rise to plastic deformation in the solid electrodeposits under consideration. Consequently, the structure refinement observed in metals being electrodeposited under action of a minor external force normal to crystallization front cannot be explained by plastic deformation in solid metal.

To verify the proposed concept that metals being 
electrodeposited pass a liquid state stage, following model experiments were performed. Copper or nickel specimens were made by electrodeposition under ordinary conditions, i. e. at an overload of $1 \mathrm{~g}$. They were subsequently placed into an electrolytic cell and subjected to a maximum attainable centrifugal force corresponding to an overload of $1256 \mathrm{~g}$ with no deposition current applied. This meant that the centrifugal force acted on a solid deposit of copper or nickel after completion of electrodeposition. No differences were found between the initial and final condition of the specimens tested.

The structure refining effect of external force action in electrodeposition of metals as revealed in this study can be explained by nucleation site number increasing due to application of pressure during solidification of the liquid metal phase. It is known that the critical nucleus size of a solid phase building in solidification of metallic liquid is inversely proportional to external pressure rise [30]. Raising external pressure must therefore result in finer solid nuclei and consequently in a finer structure of the metal being electrodeposited and solidifying from a liquid state, as observed in the experiments.

It was thus established in the experiments carried out that refinement of the intracrystalline structure and surface morphology of deposit takes place in electrodeposition of metals under exposure to a minor external force normal to crystallization front. The experimental results provided here give a proof to the existence of phenomenon of phase formation via a liquid state stage in metals being electrodeposited.

\subsection{Idea Three and Its Realization}

It is well-known that metal alloy solidification under pressure is accompanied by dislocation density rise in the solid $[41,42]$. In casting alloys, the dislocation density may be increased five to tenfold due to application of pressure during solidification [42]. If the phenomenon in point exists, then phase development in metals being electrodeposited under exposure to a minor external force must be accompanied by increased dislocation densities in solid deposits.

This assumption is based on the fact that a heavy external force large enough to give rise to plastic strain must be applied to a solid metal to increase dislocation density in it [43]. Therefore, increased dislocation density in a metal electrodeposited under exposure to a relatively low external force normal to the crystallization front will serve as a proof of the phenomenon's existence.

\subsubsection{Materials and Methods}

A centrifugal setup and electrochemical cells as described for the preceding experiment were employed to apply a minor external force normal to the crystallization front on metals being electrodeposited. The model metals were copper and nickel and the overload during electrodeposition was varied between $1 \mathrm{~g}$ (corresponding to ordinary conditions) and $872 \mathrm{~g}$ corresponding to a compressive stress of only 0.5 $\mathrm{MPa}$. As shown in Section 2.2, the latter compressive stress value is not nearly enough to give rise to plastic strain in either of said solid metals, so a dislocation density increase in the solid metal is unlikely.

The dislocation density in the electrodeposited metals was measured with an upgraded DRON-3 X-ray apparatus $\left(\mathrm{Cu}-\mathrm{K}_{\alpha}\right.$ radiation, $\left.\mathrm{U}=30 \mathrm{kV}, \mathrm{I}=20 \mathrm{~mA}\right)$ along $<111>$ direction. Since each specimen under study was crystallographically oriented [20], the dislocation density measurements were carried out for each of the various texture components in it and the average dislocation density was calculated with due account for the component proportions as recommended earlier [44-46] to improve the experimental data validity. With a view to reducing diffraction peak geometric broadening due to specimen deviations from Bragg-Brentano focusing, an X-ray bundle was passed between a pair of blades in a Soller slit system in front of the specimen.

\subsubsection{Results and Discussion}

The experimental results are a testimony of increased dislocation density in metals being electrodeposited under exposure to a minor external force (Fig. 10). Even a minimal rise in the overload from $1 \mathrm{~g}$ to $35 \mathrm{~g}$ led to an increase in the average dislocation density for copper by a factor of 3 (from $2.2 \cdot 10^{9}$ to $6.5 \cdot 10^{9} \mathrm{~cm}^{-2}$ ) and for nickel by a factor of 2.5 (from $3.1 \cdot 10^{10}$ to $7.6 \cdot 10^{10} \mathrm{~cm}^{-2}$ ). With an overload of $314 \mathrm{~g}$, the dislocation density increase reached one order of magnitude, Fig. 10; in copper electrodeposit it averaged to $2.4 \cdot 10^{10} \mathrm{~cm}^{-2}$, while in electrodeposited nickel it was at $2.8 \cdot 10^{11} \mathrm{~cm}^{-2}$.

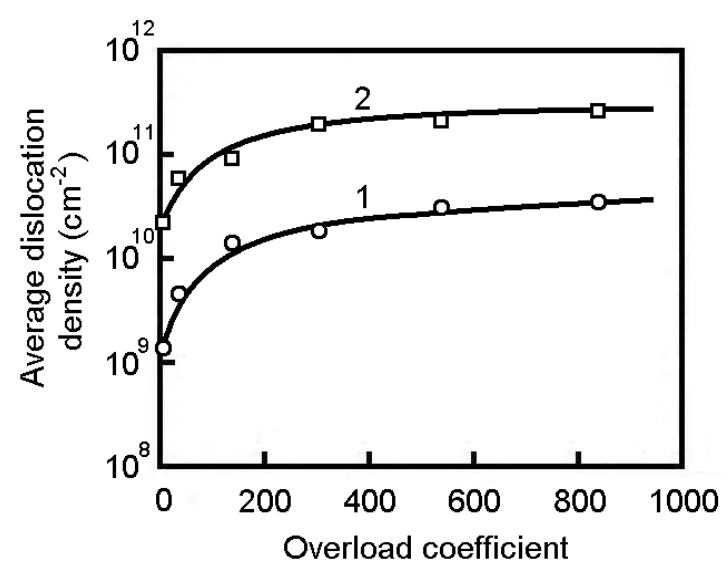

Figure 10. Average dislocation density in copper (1) and nickel (2) vs. overload coefficient in electrodeposition involving application of external force normal to the crystallization front.

Further increasing overload involved in electrodeposition to $872 \mathrm{~g}$ was accompanied by additional generation of linear defects in the emerging metal lattice. However, the rate of dislocation density elevation with overload became appreciably lower. Notably, the average dislocation density increased most rapidly over the range of smaller overloads: between $1 \mathrm{~g}$ and $140 \mathrm{~g}$ (Fig. 10). This result is in good agreement with previous experimental data showing that it was in this overload range that the electrodeposits displayed a 
dramatic rise in metal density $[17,18]$ and a considerable drop in porosity [47].

Since exposure to a minor external force during electrodeposition leads to higher dislocation density, the metal being electrodeposited cannot have stayed in a solid state at all times. The existence of an intermediate liquid state stage through which a metal goes during electrodeposition dictates its progressive solidification, which is accompanied by the occurrence of multiple linear lattice defects due to the external force.

The effect described above has two features, namely (1) nonuniform dislocation density across the various components of crystallographic texture in an electrodeposited metal, and (2) similar behavior of dislocation density in a texture component irrespective of its preferred axis. An analysis of dislocation density behavior in axial components [210] and [221] of nickel electrodeposits and in the axial component [110] of copper electrodeposits jointly with variation of dislocation density in orientationally disordered components of the deposits allows following conclusions.

First, dislocation densities in axial components are smaller than in a random component. Second, for a multicomponent axial texture, the dislocation density in the major component is lower than in a minor one. Third, linear defect generation rate in grains of any texture component is directly proportional to its degree of perfection. Fourth, dislocation density increment is highest at relatively low overloads irrespective of texture axis.

The fourth conclusion is supported by an earlier report dealing with quantitative estimation of degree of texture perfection in coatings electrodeposited in the presence of an external force [20]. The then experiments showed that considerable dissipation of axial texture occurred with increasing the overload coefficient from 1 to 314 . Notably, a minimal rise in $\mathrm{k}$ from 1 to 35 was accompanied by a strong dissipation of preferred orientation.

Consequently, one of the causes for the discovered effect of texture development suppression in a metal being electrodeposited under external force action [20] was linear defect saturation in the crystal lattice of grains disrupting their preferred orientation. Another cause of suppressed texture was possibly grain refinement leading to their misorientation.

It was thus experimentally found that dislocation density is increased when a minor external force is acting normal to the crystallization front in metal electrodeposition. The abundant generation of linear defects in the metal crystal lattice during the electrodeposition accompanied by application of a minor external force, whereas a heavy force action is required for defect generation in a solid metal, is a proof of the existence of the phenomenon in point.

\subsection{Idea Four and Its Realization}

If phase formation in metal being electrodeposited indeed passes a liquid state stage, then exposure of the solidifying metal to an external force directed at an angle to the crystallization front will bring about a number of effects. First, previously solidified particles being in a solidifying metallic liquid will move nearly parallel to the crystallization front and gradually immerse in the metallic medium until it is solidified. Such travel of solid particles along the surface of a solidifying deposit will bring about plastic deformation in its surface layers and, more specifically, to occurrence of plastic deformation bands extending through the bulk of solidifying grain aggregates, rather than following their boundaries as the case will be with deformation of a completely solid deposit.

Second, separation of a solid particle from a solidifying metal surface during electrodeposition under exposure to a force inclined to the crystallization front will leave behind, at the contact of the solid and the solidifying phase, the particle's "image" in the form of its indent including specific features of the particle's shape and morphology.

The occurrence of plastic deformation bands extending through grain aggregates due to solid particle travel along deposit surface during metal electrodeposition under exposure to a minor external force inclined to the crystallization front and the appearance of indents imaging shape and morphology of solid particles at sites of their separation from metal deposit surface during electrodeposition involving a similar force action will both be proofs of the existence of the phenomenon in point.

\subsubsection{Materials and Methods}

Exposure of metal being electrodeposited to a minor external force inclined to the crystallization front was effected by using the centrifugal unit described above. With this in view, the cathode in the electrochemical cell shown in Fig. 5 was inclined to the cell's axis. By this arrangement, the external force action on the metal being electrodeposited was no longer strictly normal to the crystallization front but directed at an angle to it. With such minor force action of solid particles on the metal being electrodeposited, no plastic deformation was to be expected in deposit surface layers if the phase formation involved no intermediate liquid phase.

The model metal to be electrodeposited was nickel selected for its yield strength, the highest of all metals under study. The SEM technique for characterization of electrodeposits surface morphology was as described above.

\subsubsection{Results and Discussion}

The experiments revealed that action of a minor force inclined to the crystallization front of the metal being electrodeposited led to plastic deformation of deposit surface layers by solid particles. This effect was manifested first of all in occurrence of plastic deformation bands extending through the bulk of grain aggregates. These deformation bands were caused by travel, along the solidifying surface of a deposit, of solid particles subjected to forces acting parallel and normal to the crystallization front at the same time.

Fig. 11 shows a characteristic feature of plastic deformation of surface layers by solid particles in a metal electrodeposited under exposure to a minor external force inclined to the crystallization front. A solid particle is seen clearly that moved along the surface of metal deposit from bottom right to top left by action of an external force. This 
travel resulted in a plastic deformation band extending through the bulk of grain aggregates (Fig. 11a).

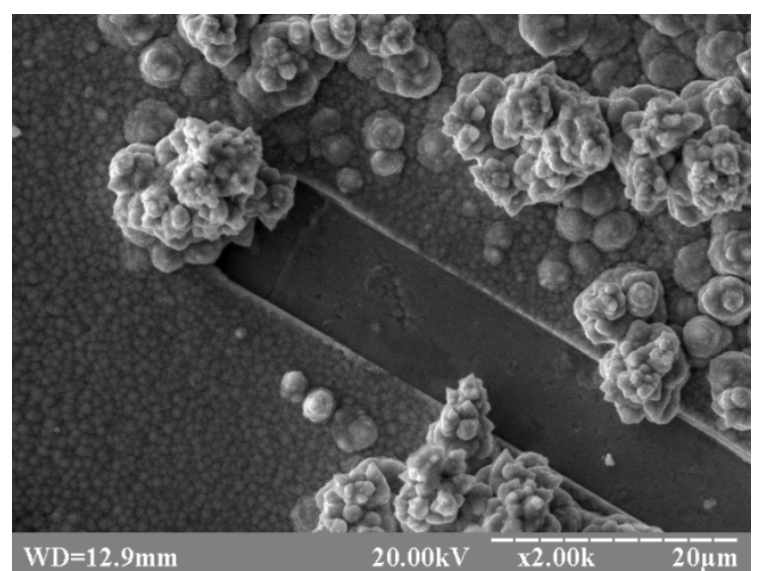

(a)

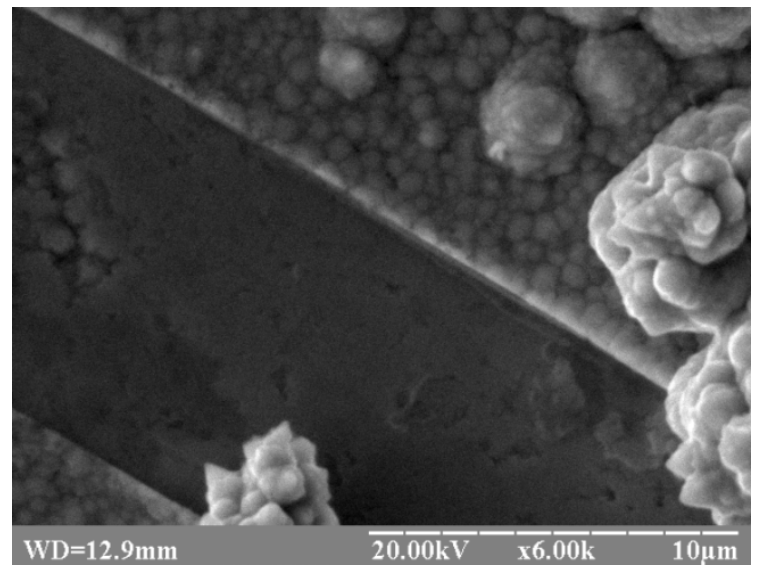

(b)

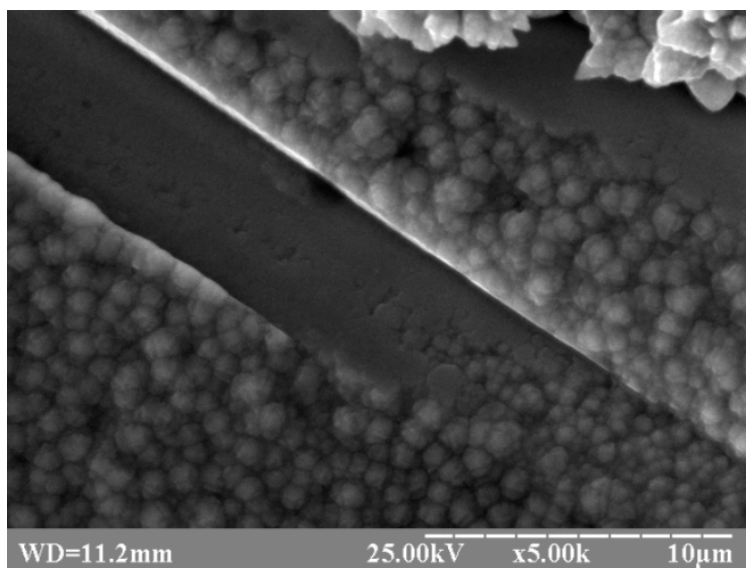

(c)

Figure 11. SEM images of nickel deposit exhibiting a plastic deformation band in its surface layers. The force was applied inclined to the crystallization front $(k=872)$; ( $a, b$ and $c$ ) images depicting respectively the final, intermediate and initial section of the deformation band.

If the particle had travelled along solid deposit's surface and gradually dug into it as it advanced, the band would have displayed a distinctive wrinkle pattern of surface layers in its final section, and grain boundaries as its edges. In the case at hand, however, the deformation band edges extend through the bulk of grain aggregates (Fig. 11b), suggesting that the deformation involved a solidifying metal and not a solid one. This conclusion is supported by the relatively smooth internal surface of the band (Fig. 11a, b) and the plastic cutting at grain aggregate tops near particle entry into the bulk of an evolving deposit (Fig. 11c).

A fact that a particle deformed a solidifying and not a solid deposit is demonstrated in Fig. 12a. The figure depicts a nickel deposit with a plastic deformation band invaded by an intragranular crack. As a rule, cracks develop during metal solidification and this particular crack came into existence after the deformation band did, as may be inferred from the observed displacement of the right band edge to the left. Hence a solidifying and not a solid metal was deformed by the particle.

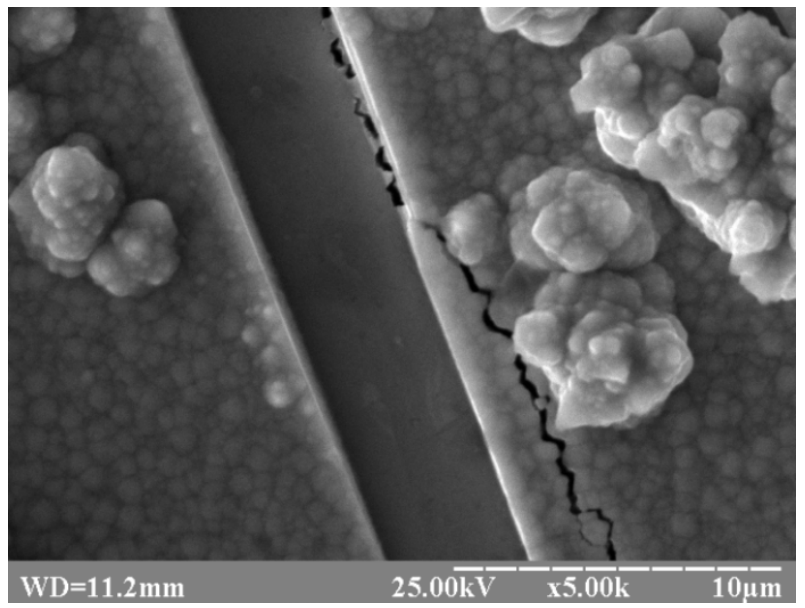

(a)

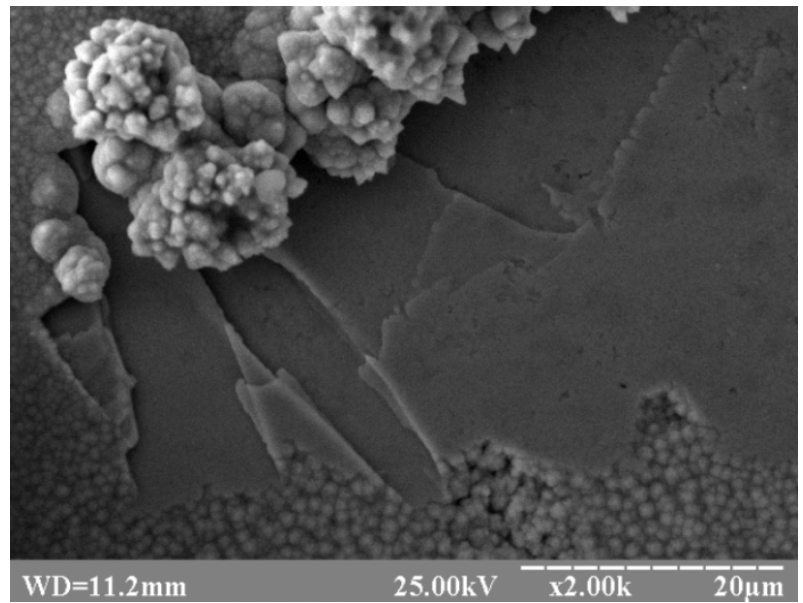

(b)

Figure 12. SEM images of nickel deposits having distinctive markers of plastic deformation by solid particles in their surface layers $(k=872)$; (a) deformation of band type, (b) deformation of area type.

An even more striking picture in support of the existence of a liquid state stage in metal electrodeposition appears as full ensembles of particles travel along the surface of developing deposit due to exposure to an external force inclined to the crystallization front. In this case, extensive plastically 
deformed areas with smoothed surface appeared due to solid particle crowds travelling along the surface of developing deposit (Fig. 12b).

With a solid metal, such surface plastic deformation clearly requires forces much in excess of the metal's yield strength. The surface layer plastic deformation effected by solid particles travelling due to exposure of the metal being electrodeposited to a minor external force inclined to the crystallization front is thus a convincing argument for the existence of the phenomenon in point.

Another brilliant experimental proof of the existence of an intermediate liquid state stage in metal electrochemical deposition is the occurrence of solid particle indents featuring particle shape and morphology and left in places where particles separated from deposit surface when the metal being electrodeposited was exposed to a minor external force inclined to the crystallization front.

An example is shown in Fig. 13 depicting a nonmetallic particle partly separated from the nickel deposit surface. Where the particle was separated, an indent is left that images the shape and morphology of the particle's separated portion. What is virtually the separated portion's mirror image frozen into the deposit surface could only be put to shape on a liquid matrix which subsequently solidified during electrodeposition.

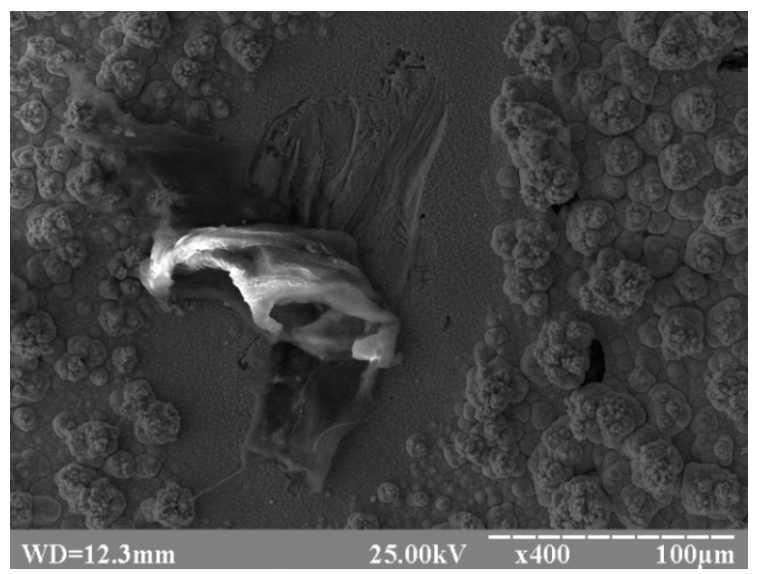

Figure 13. SEM image of nickel deposit containing a particle partly separated from deposit surface. The force was applied inclined to the crystallization front $(k=1256)$.

If no intermediate liquid state stage during phase formation in metals being electrodeposited is assumed, then no effect of this type may occur. This is because no indent of a separated particle can be shaped by "incorporation" or "embedding" of atoms (adatoms or adions) into the crystal lattice of the metal being electrodeposited.

These results agree well with previous findings of a mathematic simulation of wavelike flow in surface layers of metal being electrodeposited under exposure to an external force parallel to the crystallization front [48]. It was then found that the liquid phase of a metal being electrodeposited is a power-law fluid having distinct values of flow consistency index and flow behavior index. It is this high viscosity of the nascent, ultra-rapidly solidifying liquid metal that evidently explains the discovered experimental effects.

\section{Conclusion}

It is thus seen that the findings in experiments performed on an alternative platform and employing advancement and realization of new ideas and approaches have enabled a further step to understanding the actual processes developing in phase formation of metals being electrodeposited. Occurrence of anisotropic pattern of deposits of metals being electrodeposited, smoothing out of deposits surface morphology and reduction in their surface roughness have been established, all arising from exposure to a minor external force parallel to the crystallization front. Refinement of deposit's intracrystalline structure and surface morphology has been found for metals being electrodeposited under exposure to a minor external force normal to the crystallization front. Dislocation density rise in metals being electrodeposited under exposure to a minor external force normal to the crystallization front has been revealed. Plastic deformation in deposit surface layers effected by solid particles that travelled due to exposure of metal being electrodeposited to a minor external force inclined to the crystallization front was discovered. Solid particle indents representing the particles' shape and morphology have been detected at sites of the particles' separation from deposit surface in metals being electrodeposited under exposure to a minor external force inclined to the crystallization front. The experimental results that have come to hand prove the existence of the phenomenon of phase formation via a liquid state stage in metals being electrodeposited.

\section{References}

[1] H. Brandes und M. Volmer, "Zur theorie des kristallwachstums," Z. Phys. Chem., bd. 155 (6), ss. 466-470, 1931.

[2] I. N. Stranskii and R. Kaishev, "To the theory of crystal growth and crystal nuclei formation," Physics-Uspekhi, vol. 21 (4), pp. 408-465, 1939 (In Russian).

[3] K. M. Gorbunova and P. D. Dankov, "Elementary processes of electrocrystallization", Dokl. Akad. Nauk, vol. 48 (1), pp. 15-18, 1945 (In Russian).

[4] K. M. Gorbunova and P. D. Dankov, "Crystal-chemical theory of real growth of crystals during electrolysis," Usp. Khim., vol. 17 (6), pp. 710-732, 1948 (In Russian).

[5] W. Lorenz, "Zur theorie des elektrolytischen kristallwachstums," Z. Phys. Chem., bd. 202 (3-4), ss. 275-291, 1953.

[6] D. A. Vermilyea, "On the theory of electrolytic crystal growth," J. Chem. Phys., vol. 25 (6), pp. 1254-1263, 1956.

[7] H. Gerischer, "Zum mechanismus der elektrolytischen abscheidung und auflosung fester metalle, “ $Z$. Phys. Chem., bd. 62 (3), ss. 256-264, 1958.

[8] W. Mehl and J. O'M. Bockris, "On the mechanism of electrolytic deposition and dissolution of silver," Can. J. Chem., vol. 37 (2), pp. 190-204, 1959. 
[9] A. Milchev, Electrocrystallization. Fundamentals of Nucleation and Growth. New York: Kluwer Academic Publishers, 2002, $265 \mathrm{p}$.

[10] M. Paunovic and M. Schlesinger, Fundamentals of Electrochemical Deposition. Hoboken: WILEY-INTERSCIENCE, 2006, 375 p.

[11] E. Budevski, G. Staikov, and W. J. Lorenz, Electrochemical Phase Formation and Growth. Weinheim: WILEY-VCH, 2008, $408 \mathrm{p}$.

[12] Yu. D. Gamburg and G. Zangari, Theory and Practice of Metal Electrodeposition. New York: Springer Science, 2011, 378 p.

[13] O. B. Girin and G. M. Vorob'ev, "Change of diffraction of X-rays dissipated by metals during their electrolytic deposition," J. Phys. Chem., vol. 62 (5), pp. 1347-1349, 1988 (In Russian).

[14] O. B. Girin, "Substructure formation and texture in electrodeposits," J. Electron. Mater., vol. 24 (8), pp. 947-953, 1995.

[15] O. B. Girin, "Phenomenon of precipitation of metal being electrodeposited, occurring via formation of an undercooled liquid metal phase and its subsequent solidification. Part 1. Experimental detection and theoretical grounding," in Materials Development and Processing, vol. 8, J. V. Wood, L. Schultz, and D. M. Herlach, Eds. Weinheim: WILEY-VCH, 2000, pp. 183-188. doi: 10.1002/3527607277.ch30

[16] O. B. Girin, "Phenomenon of precipitation of metal being electrodeposited, occurring via formation of an undercooled liquid metal phase and its subsequent solidification. Part 2. Experimental verification," in Materials Development and Processing, vol. 8, J. V. Wood, L. Schultz, and D. M. Herlach, Eds. Weinheim: WILEY-VCH, 2000, pp. 189-194. doi: $10.1002 / 3527607277 . \operatorname{ch} 31$

[17] O. B. Girin, "Change of density and surface morphology of metals being electrodeposited under the action of a centrifugal force,” The Adv. Sci. J., issue 3, pp. 11-16, 2011.

[18] O. B. Girin, "Phase formation through a stage of liquid state in metallic materials being electrodeposited: recent experimental proofs,” Int. J. Mater. Sci., vol. 2 (4), pp. 108-118, 2012.

[19] O. B. Girin, "Formation of the deposits of metals being electrodeposited under the influence of a centrifugal force," The Adv. Sci. J., issue 4, pp. 51-58, 2011.

[20] O. B. Girin, "Crystallographic texture formation in metals being electrodeposited at the external force influence," Amer. J. Mater. Sci., vol. 4 (3), pp. 150-158, 2014. doi: 10.5923/j.materials.20140403.06

[21] O. B. Girin, "Phenomenon of structure formation of metals being electrodeposited via a super-cooled metal liquid, and its use for the development of advanced technologies of depositing new types of protective composite coats on canned food steel sheet," in Proc. of the 5th Int. Sci. Forum AFES. Paris: Int. Acad. of Engn, 2004, pp. 142-147.

[22] O. B. Girin, "Phase transformations in the metallic materials being electrodeposited and their application for the development of advanced technologies for anticorrosive protection of canned-food steel sheet," Mater. Sci. Forum., vol. 561-565, pp. 2369-2372, 2007.

[23] O. B. Girin, "Structure formation of metals being electrodeposited through a metal liquid as a tool for surface quality upgrading of canned food steel sheet," in Proc. of the 6th Int. Sci. Forum AFES. Hong Kong: Int. Acad. of Engn, 2005, pp. 101-103.

[24] O. B. Girin, "Phase and structure formation of metallic materials electrodeposited via a liquid state stage: new experimental proof," Defect Diffus. Forum, vol. 303-304, pp. 99-105, 2010.

[25] O. B. Girin, "Phase transformations in the metallic materials being electrodeposited," in Proc. of the 7th Int. Sci. Forum AFES "DAVOS FORUM". Davos: Int. Acad. of Engn, 2006, pp. $76-81$.

[26] O. B. Girin, "Structure features of metals obtained by electrochemical deposition and by solidification from liquid state in saturated hydrogen environment," Chem. Mater. Eng., vol. 2 (5), pp. 119-126, 2014. doi: 10.13189/cme.2014.020503

[27] A. N. Smirnov, S. L. Makulov, V. M. Safonov, and A. Yu. Tsuprun, A Large Ingot. Donetsk: Donetsk National Technical University, 2009, 278 p. (In Russian).

[28] O. B. Girin and I. D. Zakharov, "Increase of density of metals being electrodeposited under the influence of a centrifugal force," Eastern-European Journal of Enterprise Technologies, issue 5/5, pp. 4-7, 2011 (In Russian).

[29] A. M. Yampol'skii and V. A. Il'in, Brief Electroplater's Handbook. Leningrad: Engineer, 1981, 269 p. (In Russian).

[30] A. I. Batyshev, Crystallization of Metals and Alloys Under Pressure, 2nd ed. Moscow: Metallurgiya, 1990, 144 p. (In Russian).

[31] V. A. Yefimov and A. S. Eldarkhanov, Physical Methods of Influence on the Processes of Solidification of Alloys. Moscow: Metallurgiya, 1995, 272 p. (In Russian).

[32] A. N. Smirnov, V. L. Pilyushenko, S. V. Momot, and V. N. Amitan, Solidification of the Molten Metal Under External Actions. Donetsk: VIK, 2002, 169 p. (In Russian).

[33] O. B. Girin, "Substructure anisotropy in electrodeposited metals," Izv. AN SSSR Met., issue 5, pp. 122-123, 1988 (In Russian).

[34] O. B. Girin, "Texture and anisotropy of substructure in electrodeposited nickel," Izv. AN SSSR Met., issue 5, pp. 113-119, 1990 (In Russian).

[35] O. B. Girin, Yu. O. Proshenko, and E. P. Kalinushkin, "Texture of electrodeposited copper coatings as related to their substructure, granular structure and surface morphology," Texture Microstruct., vol. 34 (2-3), pp. 171-179, 2000.

[36] O. B. Girin, "A method for X-ray diffractometry analysis of polycrystalline materials having an axial texture," The Patent of Russia 1,509,697, Sept. 23, 1989 (In Russian).

[37] T. Liu, Z. C. Guo, Z. Wang, and M. Y. Wang, "Structure and corrosion resistance of nickel foils deposited in a vertical gravity field,” Appl. Surf. Sci., vol. 256, pp. 6634-6640, 2010.

[38] T. Liu, Z. C. Guo, Z. Wang, and M. Y. Wang, "Effect of gravity on the electrodeposition and characterization of nickel foils," Int. J. Miner. Metall. Mater., vol. 18 (1), pp. 59-65, 2011.

[39] V. S. Zolotorevskii, Mechanical Properties of Metals, 2nd ed. Moscow: Metallurgiya, 1983, 352 p. (In Russian). 
[40] M. Bauccio, Ed., ASM Metals Reference Book, 3rd ed. Materials Park: ASM International, 1997, 614 p.

[41] J. J. Frawley, W. F. Moore, and A. J. Kiesler, "Solidification under the application of pressure greater than atmospheric," AFS Int. Cast Metals J., vol. 5 (3), pp. 31-39, 1980.

[42] T. N. Lipchin, "Changes in the structure and properties of the alloys at the influence of pressure on the melt," Foundry Manuf., issue 7, pp. 9-10, 1985 (In Russian).

[43] I. I. Novikov, Defects in the Crystal Structure of Metals, 2nd ed. Moscow: Metallurgiya, 1975, 208 p. (In Russian).

[44] O. B. Girin and G. M. Vorob'ev, "Qualitative and quantitative assessment of substructure anisotropy in various components of texture in metallic materials," Factory Labor., vol. 49 (9), pp.55-56, 1983 (In Russian).

[45] O. B. Girin and G. M. Vorob'ev, "An X-ray method to characterize substructure anisotropy in textured materials and in various components of their texture," Apparatus and Methods of X-ray Analysis, issue 34, pp. 49-54, 1985 (In Russian).

[46] O. B. Girin, "Nonconventional X-ray diffraction techniques for coating characterization," in Solidification 1998, S. P. Marsh, J. A. Dantzig, and R. Trivedi, Eds. Warrendale: The Minerals, Metals \& Materials Society, 1998, pp. 161-169.

[47] O. B. Girin and I. M. Kuzyayev, "Experimental verification and modeling of the process of the increase of density of metals being electrodeposited at force influence," Phys. Metal. and Heat Treat. of Metals, issue 4, pp. 12-20, 2014 (In Russian).

[48] I. M. Kuzyayev and O. B. Girin, "Modeling of the process of wave-like flow of surface layers of metal being electrodeposited at external force influence," Phys. Metal. and Heat Treat. of Metals, issue 1, pp. 27-35, 2015 (In Russian). 